\title{
HYDROSTATIC PRESSURE STUDY OF MBE CdMnTe DOPED WITH BROMINE
}

\author{
J. Szczytko, D. Wasik, J. Przybytek, M. BaJ \\ Institute of Experimental Physics, Warsaw University \\ Hoża 69, 00-681 Warszawa, Poland
}

AND A. WAAG

Physikalisches Institut, Universität Würzburg, 97074 Würzburg, Germany

We present Hall effect and resistivity measurements as a function of pressure performed on MBE-grown $\mathrm{Cd}_{1-x} \mathrm{Mn}_{x} \mathrm{Te}$ (with $x=0.14$ ) layer ( $1 \mu \mathrm{m}$ ) doped with bromine. The experimental data were analysed using positive and negative $U$ model of the $\mathrm{Br}$ centres. We found that both models could reproduce the experimental points, but in the case of positive $U$ model only under assumption that the sample was completely uncompensated.

PACS numbers: 71.55.Gs, 73.61.Ga

It is known that halogen impurities in CdTe and CdTe-based ternary semiconductors create metastable centres [1-6]. Hydrostatic pressure experiments showed that in the case of CdTe these centres introduce localized levels resonant with the conduction band. However, pressure coefficients of the levels deduced from theoretical analysis were unexpectedly high. These high values could follow from the fact that the centres have so called negative $U$ character while the analysis was made under an assumption of positive $U$. Basing on old experimental data performed on $\mathrm{CdTe}$ crystals it was not possible to solve the problem: are these centres positive or negative $U$ ones? New information concerning this problem could be obtained from investigations of ternary semiconductors in which the impurity levels are close to the $\Gamma$ minimum of the conduction band or even enter the gap.

In this paper we present preliminary results of Hall effect and resistivity measurements as a function of pressure obtained on $\mathrm{Cd}_{1-x} \mathrm{Mn}_{x} \mathrm{Te}: \mathrm{Br}(x=0.14)$ doped with bromine. The aim of our work was to determine the energetic position of the $\mathrm{Br}$ level in this material as well as to study its charge state.

Bromine doped $n$-type $\mathrm{Cd}_{1-x} \mathrm{Mn}_{x}$ Te layer, $1 \mu \mathrm{m}$ thick, was grown by molecu- . lar beam epitaxy. High pressure equipment with helium gas as a pressure transmitting medium was used. An infrared GaAs LED was also mounted in our pressure cell which made the photoionization of investigated centres possible. 


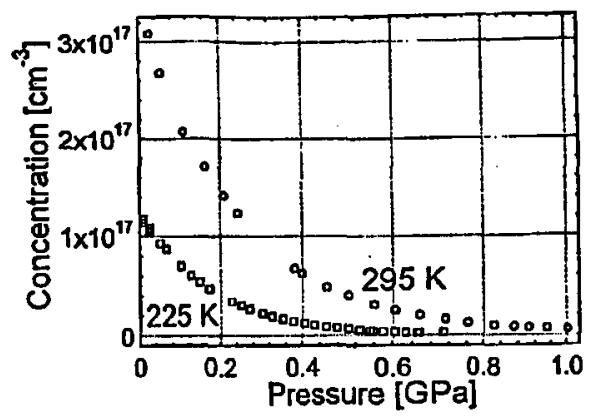

Fig. 1. Hall concentration versus pressure measured at $298 \mathrm{~K}$ (circles) and $225 \mathrm{~K}$ (squares).

The results of Hall effect measurements performed under hydrostatic pressure at room temperature are shown in Fig. 1. One can see a rapid freeze-out of electrons. This result can be explained assuming that $\mathrm{Br}$ impurity introduces a deep donor level whose energetic position is strongly irfluenced by the applied pressure. As a consequence, the impurities capture free electrons with increasing pressure leading to a change of the level and the conduction band occupations.

In contrast to this behaviour, at temperature lower than $150 \mathrm{~K}$ the free electron concentration does not depend on pressure in the dark, but depends on the pressure at which the sample was cooled down. Moreover, an illumination of the sample leads to a rapid increase in the free electron concentration up to the value of $2.27 \times 10^{18} \mathrm{~cm}^{-3}$ (independent of the pressure at which the sample was cooled down and then illuminated), which is much higher than that measured at $p=0$ and $T=300 \mathrm{~K}\left(2.23 \times 10^{17} \mathrm{~cm}^{-3}\right)$. The above low temperature experiments (see Fig. 2) reveal the metastable character of the investigated level which is in close analogy with effects observed for DX-like centres in III-V and II-VI compounds [6].

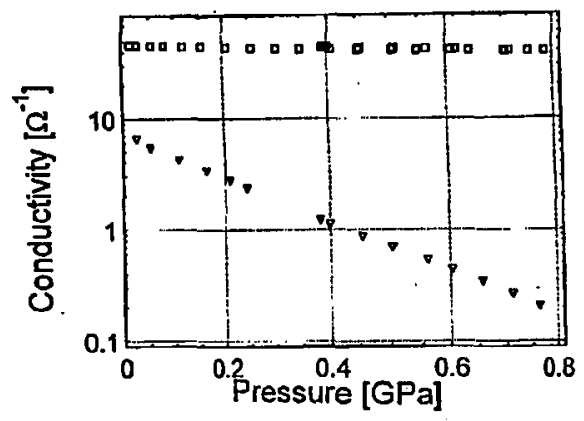

Fig. 2. Conductivity as a function of pressure applied at room temperature (triangles) and then released at $T=77 \mathrm{~K}$ after illumination (squares). 


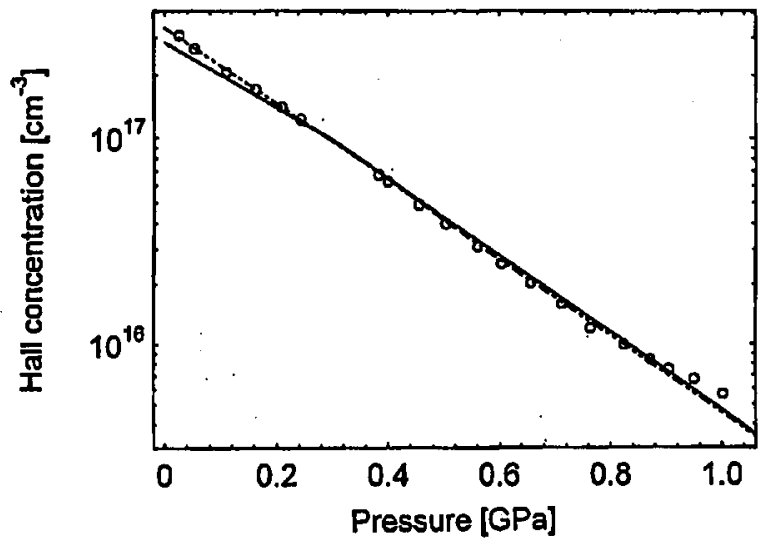

Fig. 3. Hall concentration versus pressure measured at $T=298 \mathrm{~K}$. The theoretical calculations: solid (dashed) line presents the negative (positive) $U$ model.

In order to determine the thermal ionization energy, $E_{\mathrm{Br}}$ of the $\mathrm{Br}$ level and its pressure shift we used two models of the level charge state: i.e. positive $U$ and negative $U$. In both cases we assumed that: (1) the Br level is in thermal equilibrium with the conduction band at temperatures greater than $150 \mathrm{~K}$; (2) it has $\delta$-like density of states, (3) the concentration of $\mathrm{Br}$ impurities is equal to $N_{\mathrm{Br}}=n(77 \mathrm{~K})+N_{\mathrm{A}}$, where $n(77 \mathrm{~K})=2.27 \times 10^{18} \mathrm{~cm}^{-3}$, i.e. the value of the electron concentration measured at $77 \mathrm{~K}$ after illumination and $N_{\mathrm{A}}$ is the acceptor concentration.

In the case of positive $U$ model, impurity level is a donor $\mathrm{D}(0 /+)$ and the charge neutrality equation is the following: $n+N_{\mathrm{A}}=N_{\mathrm{Br}}\left(N_{\mathrm{Br}}+=N_{\mathrm{Br}} /\{1+\right.$ $\left.2 \exp \left[E_{\mathrm{F}}-E(p)\right] / k T\right\}$, where $E_{\mathrm{F}}-$ Fermi energy, $E(p)=E_{\mathrm{Br}}+\left(\delta E_{\mathrm{Br}} / \delta p\right) p$. The thermal ionization energy $E_{\mathrm{Br}}$ and its pressure coefficient $\delta E_{\mathrm{Br}} / \delta p$ were treated as fitting parameters. It appeared that good agreement between the calculation and measured data (see Fig. 3) could be obtained only under the assumption that the sample was practically uncompensated $\left(N_{\mathrm{A}}<10^{16} \mathrm{~cm}^{-3}\right)$. In this case $E_{\mathrm{Br}}=60 \mathrm{meV}$ below the $\Gamma$ conduction band minimum and $\mathrm{Br}$ level pressure coefficient was very high $\delta E_{\mathrm{Br}} / \delta p=-230 \mathrm{meV} / \mathrm{GPa}$ - the value consistent with old data obtained for bulk samples [2].

The next model (i.e. negative U) assumes that the occupying of the $\mathrm{Br}$ level $\mathrm{D}(-/+)$ means trapping two electrons. The charge balance equation was in the form $n+N_{\mathrm{A}}+N_{\mathrm{Br}^{-}}=N_{\mathrm{Br}+}$ (where $N_{\mathrm{Br}^{-}}+N_{\mathrm{Br}^{+}}+N_{\mathrm{Br}^{0}}=N_{\mathrm{Br}}$ ). It appeared that the best fits made in this model as well as in the positive $U$ one described above, are of the same quality, however negative $U$ model does not need an assumption of zero compensation and gives other values of the considered level parameters, i.e. $E_{\mathrm{Br}}=\left(E_{\mathrm{Br}}^{0 /+}+E_{\mathrm{Br}}^{-/ 0}\right) / 2=8 \mathrm{meV}, \delta E_{\mathrm{Br}} / \delta p=-120 \mathrm{meV} / \mathrm{GPa}$. Comparing both models it seems that positive $U$ model is less realistic due to assumption of zero compensation (which is difficult to understand taking into account a small value of measured mobility $\approx 100 \mathrm{~cm}^{2} /(\mathrm{V} \mathrm{s})$ and because it gives enormously high values of pressure coefficients. The problem of the compensation and high values 
of pressure coefficients, reported in the literature earlier could be now overcome in the framework of negative $U$ model. However, we would like to conclude that more studies on ternary CdMnTe semiconductors with smaller content of Mn are needed to solve the problem of the $\mathrm{Br}$ level charge state. From our analysis it follows that the best sample is in which at ambient pressure the level is slightly above the bottom of the conduction band and under pressure enters the gap (i.e. $\mathrm{Cd}_{1-x} \mathrm{Mn}_{x} \mathrm{Te}: \mathrm{Br}$ with $x$ about $7 \%$ ).

This work has been partially supported by the State Committee for Scientific Research (Republic of Poland).

\section{References}

[1] G.W. Isler, J.A. Kafalas, A.J. Strauss, Solid State Commun. 10, 619 (1972).

[2] M. Baj, L. Dmowski, M. Konczykowski, S. Porowski, Phys. Status Solidi A 33, 421 (1976).

[3] B.C. Burkey, R.P. Khosla, J.R. Fisher, D.J. Losee, J. Appl. Phys. 47, 1095 (1976).

[4] K. Khachaturyan, M. Kamińska, E.R. Weber, P. Becla, Phys. Rev. B 40, 6304 (1989).

[5] T. Wojtowicz, G. Karczewski, N.G. Semaltianos, S. Koleśnik, I. Miotkowski, M. Dobrowolska, J.K. Furdyna, Mater. Sci. Forum 143-147, 1203 (1994).

[6] S. Scholl, J. Gerschütz, H. Schäfer, F. Fischer, A. Waag, G. Landwehr, Solid State Commun. 91, 491 (1994). 